\title{
Glycyrrhizae Radix et Rhizoma Processed by Sulfur Fumigation Damaged the Chemical Profile Accompanied by Immunosuppression and Liver Injury
}

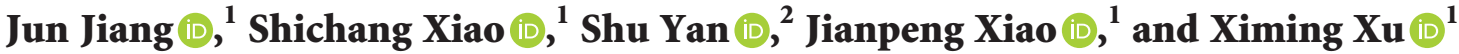 \\ ${ }^{1}$ School of Pharmacy, Jiangsu University, 301 Xuefu Road, Zhenjiang 212013, Jiangsu Province, China \\ ${ }^{2}$ ADR Monitoring Center, Zhenjiang Food and Drug Supervision and Inspection Center, 62 ${ }^{*}$ Nanxu Road, Zhenjiang 212000, \\ Jiangsu Province, China
}

Correspondence should be addressed to Shu Yan; 31491146@qq.com and Ximing Xu; xmxu@ujs.edu.cn

Received 1 October 2019; Accepted 5 November 2019; Published 6 February 2020

Guest Editor: Di Jiang

Copyright (c) 2020 Jun Jiang et al. This is an open access article distributed under the Creative Commons Attribution License, which permits unrestricted use, distribution, and reproduction in any medium, provided the original work is properly cited.

Glycyrrhizae Radix et Rhizoma (GRER) has been used as a medicinal plant and dietary supplements for its beneficial effect in immunomodulatory effects. Sulfur fumigation (SF) processing was widely used in the storage and maintenance of Chinese medicine because of its convenience and cheapness. However, the disadvantage of SF has been reported, but the systematic study of SF on GRER was deficient. In this paper, the active ingredients, sulfur-fumigated products, immunomodulatory effect, and liver injury of SF-GRER were studied. After SF, the liquiritin decreased from $4.49 \pm 0.03 \mathrm{mg} / \mathrm{g}$ to $3.94 \pm 0.08 \mathrm{mg} / \mathrm{g}(P<0.01)$. Compared with the NSF-GRER group, the SF-GRER group showed a decreased immunoregulation in the thymus index, spleen index, and serum IL-6 and SOD levels $(P<0.05)$. After 2 weeks of continuous intragastric administration of SF-GRER in healthy mice, the level of serum aspartate aminotransferase (AST) significantly increased $(P<0.05)$ and the area of liver lesion significantly increased compared with the NSF-GRER $(P<0.05)$ group. The sulfonated products $(\mathrm{m} / z, 631.13)$ corresponding to liquiritin apioside $(\mathrm{m} / z, 551.17)$ and isoliquiritin apioside $(\mathrm{m} / z, 551.17)$ were screened out in SF-GRER by using UPLC-Orbitrap-MS. The sulfonated products provided in this paper were discovered for the first time and could be powerfully applied for the identification of SF-GRER. SF destroyed the chemical composition of GRER, inhibited immunoregulation, and induced liver injury. The feasibility of this processing method needs to be reconsidered.

\section{Introduction}

Glycyrrhizae Radix et Rhizoma (GRER) has been used as a medicinal plant and dietary supplements since ancient times in China, Japan, Italy, United States, and other different countries and regions [1-4]. Modern research studies showed that GRER had extensive pharmacological effects in antispasmodic, antidiabetic, antiosteoporosis, antidepressive, antitussive and expectorant, hepatoprotective, and memory-enhancing [5-11]. The major bioactive components of GRER were saponins, flavonoid glycosides, and free phenolic compounds [12]. Approximately $250 \mathrm{com}-$ pounds have been reported from GRER, and more than 151 compounds in GRER have been determined up to present [13]. In the clinical practice of traditional Chinese medicine, there was a saying of "Ten prescriptions and Nine GRER." Therefore, the clinical demand of GRER is very large.

In recent years, sulfur fumigation (SF) has been used to replace traditional sun-drying for various Chinese herbal medicines to prevent pest infestation, mold, browning, and bacterial contamination in a cheap and convenient manner. The raw materials were usually stacked together, and a pot of burning sulfur was placed at the bottom and fumigated in a closed space for 12-24 hours. There have been many reports of sulfur-fumigated traditional Chinese medicine, including ginseng [14], Angelicae sinensis Radix [15], Chrysanthemum morifolium flowers [16], Ophiopogonis Radix [17], Atractylodes macrocephala Koidz. [18], Fritillaria thunbergii Miq. [19], Radix Paeoniae Alba [20], Smilacis Glabrae Rhizoma [21], Gastrodia Rhizoma [22], and lots of others. Numerous 
studies have shown that SF-induced dramatic changes in chemical profiles which makes the safety and effectiveness of sulfur-fumigated traditional Chinese medicine have hidden dangers [23]. Therefore, the investigations of SF on chemical profiles, bioactivities, and toxicity of Chinese medicine are urgently needed for reevaluating this potentially harmful processing method. However, the effects of SF on active components, immunomodulation, and liver function of GRER and how to identify sulfur-fumigated GRER are still deficient.

In the present study, firstly, the content changes of main components in GRER before and after SF were detected by HPLC. Secondly, the immunosuppressive mice model and healthy mice model were, respectively, applied to investigate the effects of SF on immunoregulation and liver injury of GRER. Finally, liquid chromatography coupled with linear ion trap hybrid orbitrap mass spectrometry (UPLC-Orbitrap-MS) method was applied to screen the characteristic products in sulfur-fumigated GRER.

\section{Materials and Methods}

2.1. Ethics Statement. All animal experiments strictly comply with the Guidelines for Animal Experimentation of Jiangsu University (Zhenjiang, China), and the protocol was approved by the Animal Ethics Committee of this institution.

2.2. Materials and Chemicals. GRER was purchased from Gansu Province (Lanzhou Minxian Special Products Co., Ltd.) and identified as the dried roots and rhizomes of Glycyrrhiza uralensis Fisch. by Professor Wu Chengying in Jiangsu Academy of Traditional Chinese Medicine. Liquiritin (no. K186728, purity $>99.0 \%$ ) was purchased from Xi'an Kaili Bioengineering Co., Ltd (Xi'an, China). Sublimated sulfur was purchased from Chemical Reagents Co., Ltd. of China Pharmaceutical Group (Shanghai, China). The chromatographic distilled water was made in our laboratory. Chromatographic pure acetonitrile and methanol were purchased from Shanghai Xingke High Purity Solvent Co., Ltd (Shanghai, China). Analytical pure sodium chloride, cyclophosphamide, formaldehyde, and sodium heparin were purchased from Titan Technology Co., Ltd. (Shanghai, China).

\subsection{Preparation of Sulfur-Fumigated and Nonsulfur-Fumi-} gated Samples. Sulfur-fumigated GRER (SF-GRER) was prepared according to the method used in literature [24]. The appropriate amount of nonsulfur-fumigated GRER (NSF-GRER) and SF-GRER was weighed and extracted twice with 10 times of water. Combined with the two extracts and concentrating to $100 \mathrm{~mL}$ under vacuum, the freeze-dried powder was obtained. For the accuracy of the results, 5 batches of SF-GRER and NSF-GRER were prepared in parallel. $0.2 \mathrm{~g}$ of freeze-dried powder of NSF-GRER and SFGRER were separately weighed in a conical bottle, and $50 \mathrm{~mL}$ methanol was added immediately. After $30 \mathrm{~min}$ of ultrasound-assisted extraction, the membrane-crossed $(0.22 \mu \mathrm{m})$ extracting solution was used for HPLC-DAD and OrbitrapMS analysis.
2.4. Animals and Administration. Male KM mice $(20 \pm 2 \mathrm{~g})$ were supplied by Laboratory Animal Center of Jiangsu University. All animals were housed under $20-25^{\circ} \mathrm{C}$ with a relative humidity of $40-70 \%$. All the experimental protocols were approved by the Animal Ethics Committee of Jiangsu University. Animals were divided into two parts. Part I includes mice divided into 6 groups $(n=10)$, and they were normal control group (CON, normal saline, i.g), model group MOD, after continuous intraperitoneal injection of cyclophosphamide $(150 \mathrm{mg} / \mathrm{kg})$ for 3 days, SF-GRER lowdose group (SF-L, $2 \mathrm{~g} / \mathrm{kg} /$ day, i.g), SF-GRER high-dose group (SF-H, $4 \mathrm{~g} / \mathrm{kg} /$ day, i.g), NSF-GRER low-dose group (NSF-L, $2 \mathrm{~g} / \mathrm{kg} /$ day, i.g), and NSF-GRER high-dose group (NSF-H, $4 \mathrm{~g} / \mathrm{kg} /$ day, i.g). Part II includes healthy mice separately gavaged in NSF-GRER or SF-GRER at high or low doses (the same dose as part I, $n=10$ ) for 14 consecutive days to investigate the effects of SF-GRER on liver function.

2.5. Collection and Treatment of Mice Samples. The body weight of mice in each group of part I was recorded daily. After 10 days of continuous administration, the whole blood of mice in each group was obtained by eyeball extraction and the serum was separated by centrifugation. Serum SOD and IL-6, thymus index, and spleen index of every group in part I were detected, respectively. For part II, after 14 days of continuous administration, the serum AST was detected and their liver were obtained and fixed by $10 \%$ formalin solution. The liver injury was assessed by HE staining and percentage of lesions.

2.6. Sample Analysis. The HPLC coupled with DAD and LCsolution (LC-AT $\mathrm{SR}_{\mathrm{SR}}$ Shimadzu, Japan) was used for the content determination of liquiritin. The chromatographic separation was operated with an Agilent Zorbax SB-C 18 column $(4.6 \mathrm{~mm} \times 250 \mathrm{~mm}, 5 \mu \mathrm{m})$ under column temperature $40^{\circ} \mathrm{C}$, detection wavelength $275 \mathrm{~nm}$, flow rate $1.0 \mathrm{~mL} /$ min, and injection volume $20 \mu \mathrm{L}$. The mobile phase was acetonitrile (A) and water (B) under a gradient elution (0-15 min, 18\% A; 15-20 min, 18-60\% A; 20-30 min, 60\% A; 30-40 min, 60-18\% A; and 40-42 min, 18\% A; Figures 1(a) and 1(b)). UPLC-Orbitrap-MS was equipped with an electrospray ionization source under positive mode. Chromatographic separation was performed using a Dionex U3000 UPLC system with a Phenomenex Kinetex $C_{18}$ column $(2.1 \mathrm{~mm} \times 100 \mathrm{~mm}, 2.6 \mu \mathrm{m})$. The column temperature was set at $30^{\circ} \mathrm{C}$. The mobile phase consisted of solvent $\mathrm{A}$ (acetonitrile) and solvent B ( $0.1 \%$ formic acid in water) with gradient program: 0 to $5 \mathrm{~min}, 5 \% \mathrm{~A} ; 5$ to $15 \mathrm{~min}, 5-35 \% \mathrm{~A}$; 15 to $20 \mathrm{~min}, 35-85 \% \mathrm{~A} ; 20$ to $28 \mathrm{~min}, 85-5 \% \mathrm{~A}$; and 28 to $30 \mathrm{~min}, 5 \% \mathrm{~A}$ (Figures $1(\mathrm{c})$ and $1(\mathrm{~d})$ ). The flow rate was $0.25 \mathrm{~mL} / \mathrm{min}$, and the sampling volume was $5 \mu \mathrm{L}$. For the MS conditions, the spray voltage was $3.5 \mathrm{kV}$, the heated capillary temperature was $300^{\circ} \mathrm{C}$, the ESI probe temperature was $350^{\circ} \mathrm{C}$, the flows of sheath gas and auxiliary gas were 40 units and 15 units, respectively, the scan range was $500-1800 \mathrm{~m} / z$ with the Orbitrap analyzer, and target ions selected for fragmentation were obtained by dynamic exclusion for $20 \mathrm{~s}$. All the data analysis was performed using Xcalibur 2.2 SP1 software (Thermo Fisher Scientific, Inc., Bremen, Germany). 


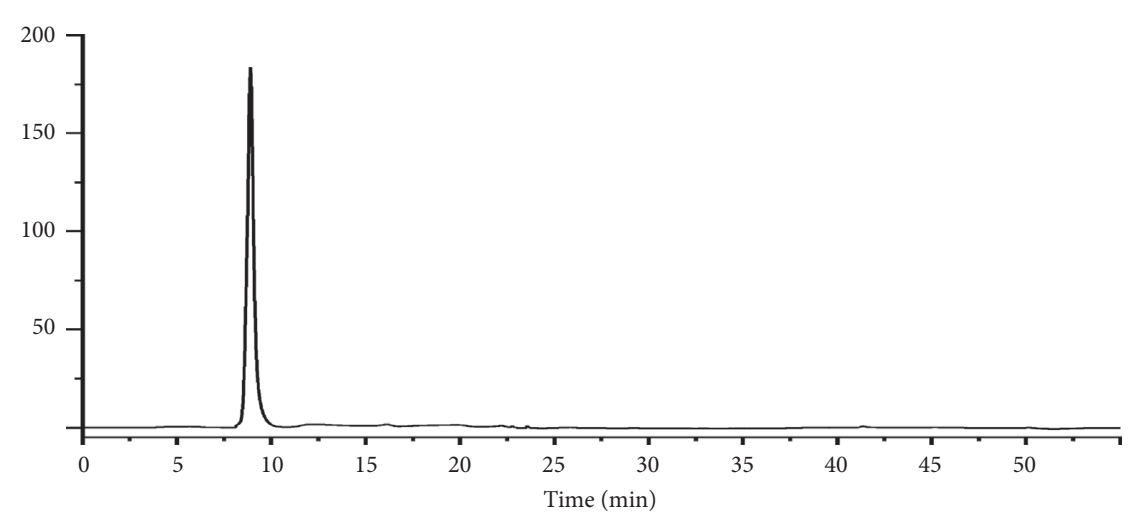

(a)

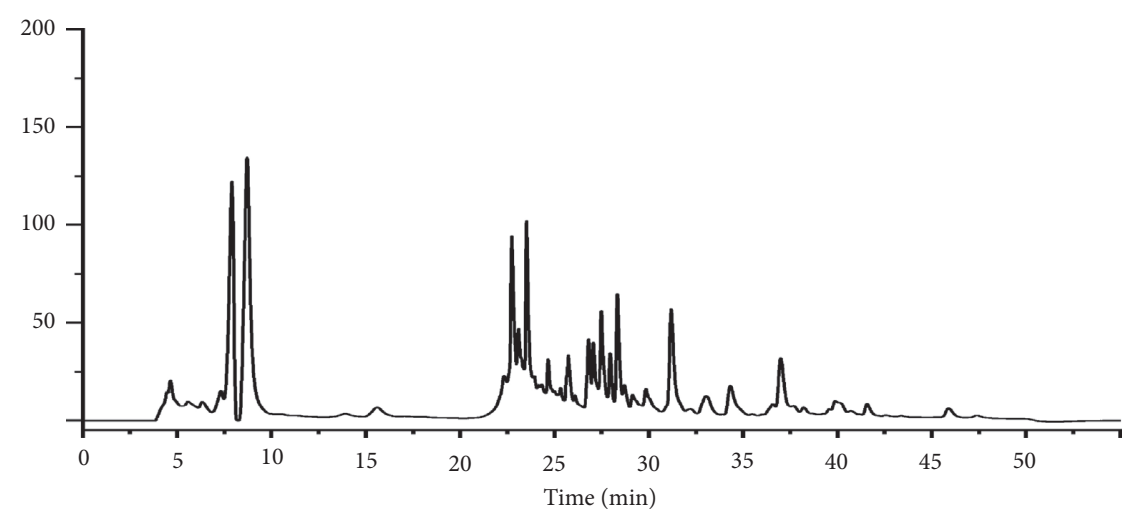

(b)

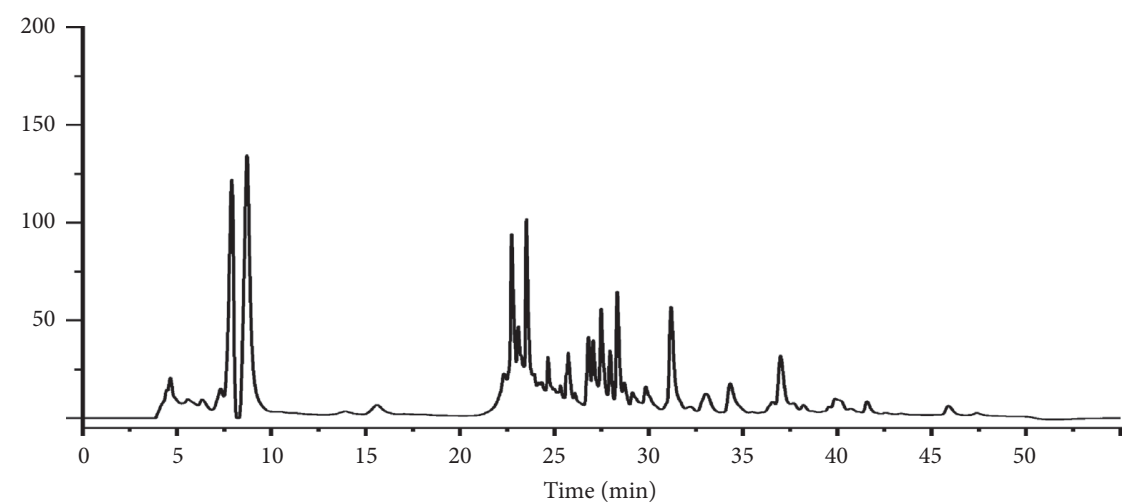

(c)

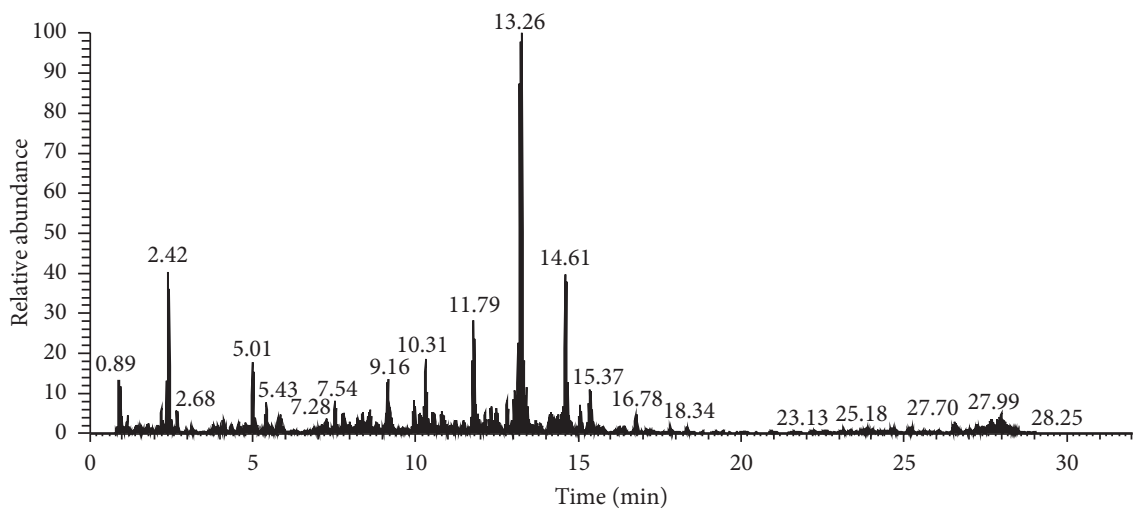

(d)

Figure 1: Continued. 


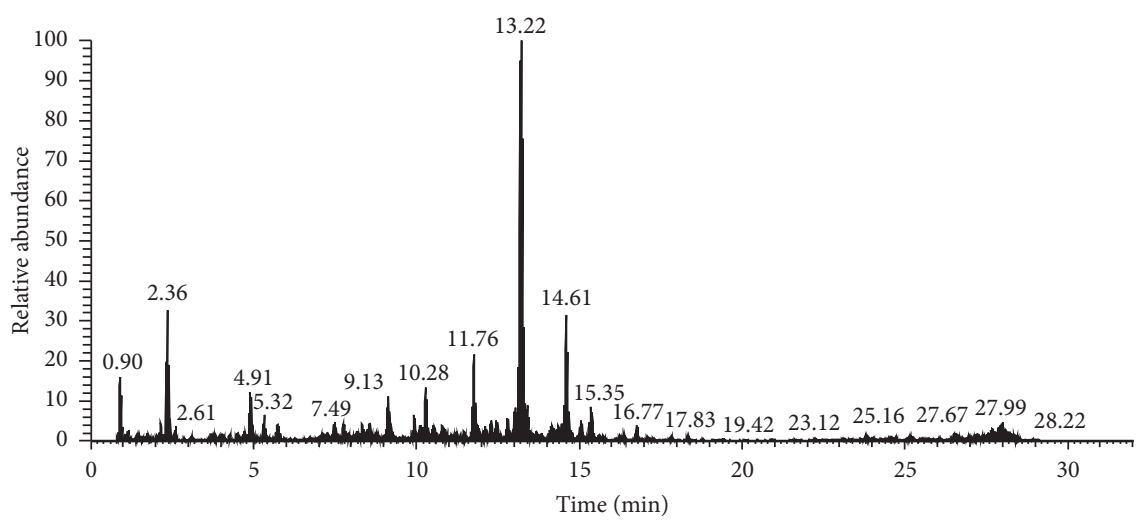

(e)

FiguRE 1: HPLC-DAD and UPLC-Orbitrap-MS chromatogram: (a) chromatography of liquiritin standard solution; (b) chromatography of freeze-dried powder of NSF-GRER extract; (c) chromatography of freeze-dried powder of SF-GRER extract; (d) total ion chromatography of NSF-GRER; (e) total ion chromatography of SF-GRER.

TABLE 1: Recovery of liquiritin in NSF-GRER and SF-GRER by HPLC.

\begin{tabular}{|c|c|c|c|c|c|c|}
\hline Samples & Original quantity (mg) & Added & Determined & Recovery & Average (\%) & RSD \\
\hline \multirow{5}{*}{ NSF } & 0.449 & 0.400 & 0.853 & 101.10 & 100.27 & 1.08 \\
\hline & 0.459 & 0.400 & 0.857 & 99.55 & & \\
\hline & 0.447 & 0.400 & 0.846 & 99.83 & & \\
\hline & 0.455 & 0.400 & 0.862 & 101.70 & & \\
\hline & 0.453 & 0.400 & 0.849 & 99.15 & & \\
\hline \multirow{5}{*}{ SF } & 0.400 & 0.400 & 0.795 & 98.70 & 100.37 & 1.47 \\
\hline & 0.402 & 0.400 & 0.80465 & 100.65 & & \\
\hline & 0.391 & 0.400 & 0.801 & 102.55 & & \\
\hline & 0.401 & 0.400 & 0.798 & 99.35 & & \\
\hline & 0.397 & 0.400 & 0.799 & 100.60 & & \\
\hline
\end{tabular}

2.7. Biochemical Indices and Pathological Evaluation. The thymus and spleen index were calculated as follows: spleen (thymus) index = the spleen (thymus) mass (mg)/the body weight $(\mathrm{g})$. Blood samples were collected from eyeballs at intervals of one hour after the last administration. The blood samples were placed in a $2 \mathrm{~mL}$ centrifugal tube containing $20 \mu \mathrm{L}$ of $5 \%$ heparin sodium. The supernatant was centrifuged at $3700 \mathrm{rpm}$ for $10 \mathrm{~min}$ and frozen $\left(-80^{\circ} \mathrm{C}\right)$ in the centrifugal tube. Serum SOD, IL-6, and AST were determined by ELISA [25]. HE staining sectioning $(2 \mu \mathrm{m})$ was read under optical microscope (OLYMPUS BX41, Japan) [26]. The main examination of the liver was (1) whether there is hepatocyte degeneration (steatosis and edema), (2) whether there is hepatocyte degeneration and necrosis, (3) whether there is inflammatory cell infiltration in the liver lobule, (4) whether there is inflammation or fibrous tissue proliferation in the portal area, and (5) whether there is atrophy of hepatocyte and expansion of hepatic sinuses. Five visual fields were selected for each slice, and then the average percentage of lesion in different groups was calculated.

2.8. Statistical Analysis of Data. Statistical analyses were conducted using one-way ANOVA (GraphPad Prism 5.0) followed by the Tukey test for comparing all pairs of columns. Data were presented as mean value \pm SD. The $P$ value $<0.05$ was considered as statistically significant.
TABLE 2: Liquiritin in GRER before and after sulfur fumigation $(n=3)$.

\begin{tabular}{lcccc}
\hline \multirow{2}{*}{ No. } & \multicolumn{2}{c}{ NSF $(\mathrm{mg} / \mathrm{g})$} & \multicolumn{2}{c}{ SF $(\mathrm{mg} / \mathrm{g})$} \\
& Content & Average content & Content & Average content \\
\hline 1 & 4.524 & & 3.864 & \\
2 & 4.472 & & 3.934 & \\
3 & 4.446 & $4.49 \pm 0.03$ & 3.884 & $3.94 \pm 0.08^{\# \#}$ \\
4 & 4.485 & & 3.947 & \\
5 & 4.506 & 4.064 & \\
${ }_{\# \# \text { Compared with NSF, the content decreased significantly }(P<0.01) .}$
\end{tabular}

\section{Results}

3.1. Methodological Validation. The standard solution was determined according to the chromatographic conditions under item "HPLC-DAD analysis." The peak area of liquiritin $(Y)$ was linearly regressed by sample concentration $(X)$. The results showed that the linear relationship of liquiritin was fine in the range of $20-200 \mu \mathrm{g} / \mathrm{mL}$. The regression equation was $Y=42438 X-232006, r^{2}=0.9998$. The RSD of intra- and interday precision was $0.32 \%$ and $0.51 \%$, indicative of a high precision. The RSD of repeatability in SFGRER and NSF-GRER was $0.67 \%$ and $1.98 \%$, respectively. The detection limit (LOD) and quantitative limit (LOQ) of liquiritin was $0.06 \mu \mathrm{g} / \mathrm{mL}$ and $0.21 \mu \mathrm{g} / \mathrm{mL}$, respectively. The 


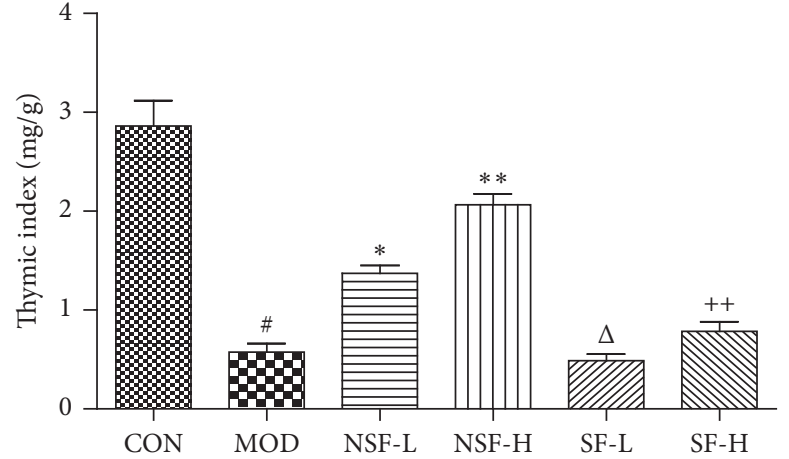

(a)

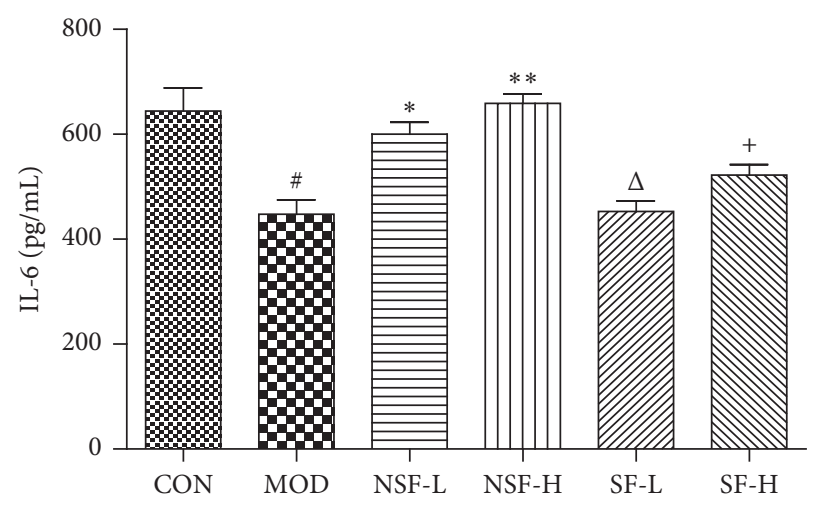

(c)

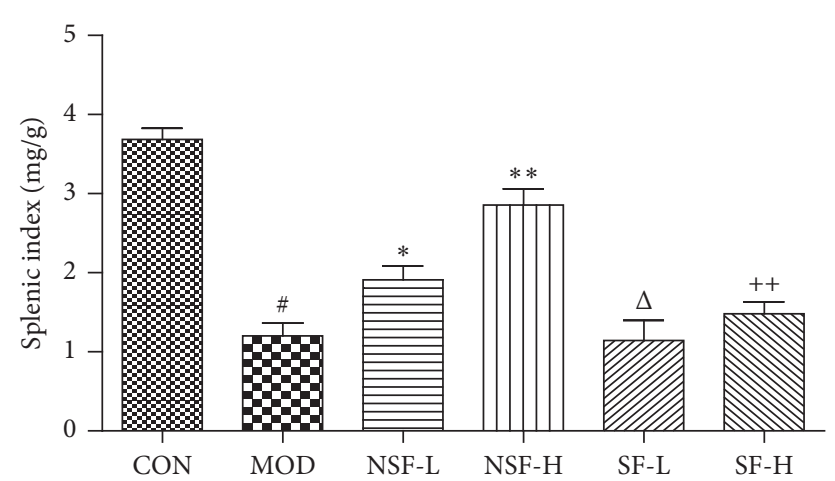

(b)

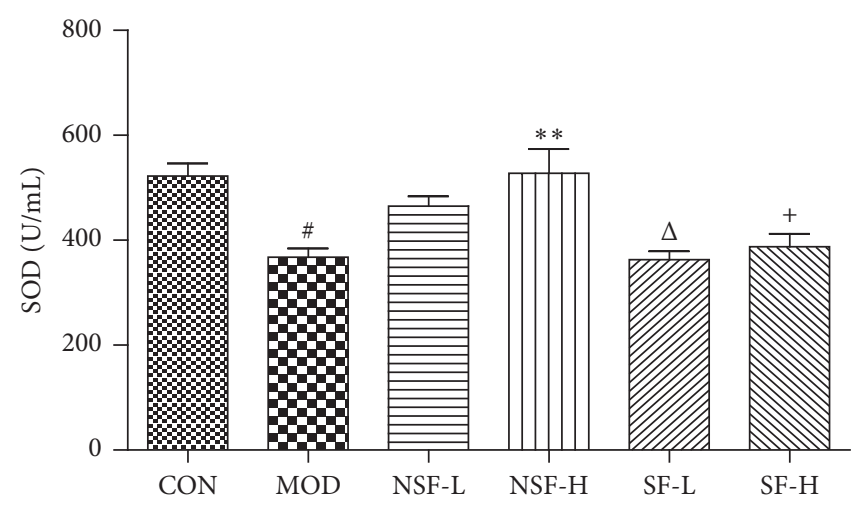

(d)

FIGURE 2: Immunoregulation of GRER before and after sulfur fumigation $(\bar{x} \pm \mathrm{SD}, n=10) .{ }^{*}$ Compared with $\mathrm{CON}, P<0.01 ;{ }^{*}$ compared with MOD, $P<0.05 ;{ }^{* *}$ compared with MOD, $P<0.01 ;{ }^{\Delta}$ compared with NSF-L, $P<0.05 ;{ }^{+}$compared with NSF-H, $P<0.05 ;{ }^{++}$compared with NSF-H, $P<0.01$.

recovery rates of liquiritin in SF-GRER and NSF-GRER were $100.37 \%$ and $100.27 \%$, respectively (Table 1 ). The method had superior stability, reproducibility, and recovery which ensured the accuracy of the quantitative results.

3.2. Liquiritin before and after Sulfur Fumigation. The liquiritin in NSF-GRER was $4.49 \pm 0.03 \mathrm{mg} / \mathrm{g} \quad(0.449 \%)$. Compared with NSF-GRER, the liquiritin in SF-GRER decreased significantly to $3.94 \pm 0.08 \mathrm{mg} / \mathrm{g} \quad(0.394 \%$, $P<0.01)$. The liquiritin in GRER before and after sulfur fumigation was shown in Table 2. Our results showed that liquiritin was destroyed or transformed into other structures during sulfur fumigation.

3.3. Immunomodulatory Effects before and after Sulfur Fumigation. Compared with the healthy mice, the thymus index $(0.58 \pm 0.15 \mathrm{mg} / \mathrm{g})$, spleen index $(1.20 \pm 0.36 \mathrm{mg} / \mathrm{g})$, serum IL-6 $(448.00 \pm 54.40 \mathrm{pg} / \mathrm{mL})$, and SOD $(367.50 \pm 33.67 \mathrm{U} /$ $\mathrm{mL})$ levels of the model group were significantly decreased by cyclophosphamide $(P<0.01)$. Under the intervention of NSFGRER (low and high dose), the immunosuppression induced by cyclophosphamide could be reversed and the thymus index $(1.37 \pm 0.16$ and $2.06 \pm 0.19 \mathrm{mg} / \mathrm{g})$, spleen index $(1.91 \pm 0.39$ and $2.86 \pm 0.35 \mathrm{mg} / \mathrm{g})$, serum IL-6 $(600.11 \pm 46.44$ and $658.77 \pm 35.99 \mathrm{pg} / \mathrm{mL})$, and SOD levels $(465.00 \pm 37.97$ and $527.50 \pm 92.11 \mathrm{U} / \mathrm{mL})$ were significantly increased $(P<0.05)$.
However, with the intervention of SF-GRER, the indicators showed an upward trend but no significant difference. It is worth noting that, compared with the NSF-GRER group, the SF-GRER group showed a significant decrease in immunoregulation $(P<0.05)$. Detailed results are shown in Figure 2.

3.4. Effects on Liver Function. After 2 weeks of continuous intragastric administration of SF-GRER and NSF-GRER in healthy mice, the level of AST increased significantly in SFGRER mice $(P<0.05$, Figure 3(a)). Therefore, we suspected that SF-GRER may induce liver damage in healthy mice $[27,28]$. Subsequent pathological studies showed that SFGRER significantly increased the area of liver lesion compared with the same dose of NSF-GRER $(P<0.05$, Figure 3(b)).

3.5. Screening of Sulfur-Fumigated Characteristic Products. UPLC-Orbitrap-MS has been widely used in the identification of characteristic components of traditional Chinese medicine [29, 30]. According to related literature $[2,4,13,31-34]$ and the transformation rule before and after $\mathrm{SF}$, the molecular weight ranging from 500 to $1800(\mathrm{~m} / z)$ was screened one by one under the UPLC-Orbitrap-MS system. Fortunately, liquiritin apioside (LA, $m / z, 551.17$ ) and isoliquiritin apioside (ILA, $m / z$, 551.17) not only had been found in NSF-GRER, their corresponding sulfonated 


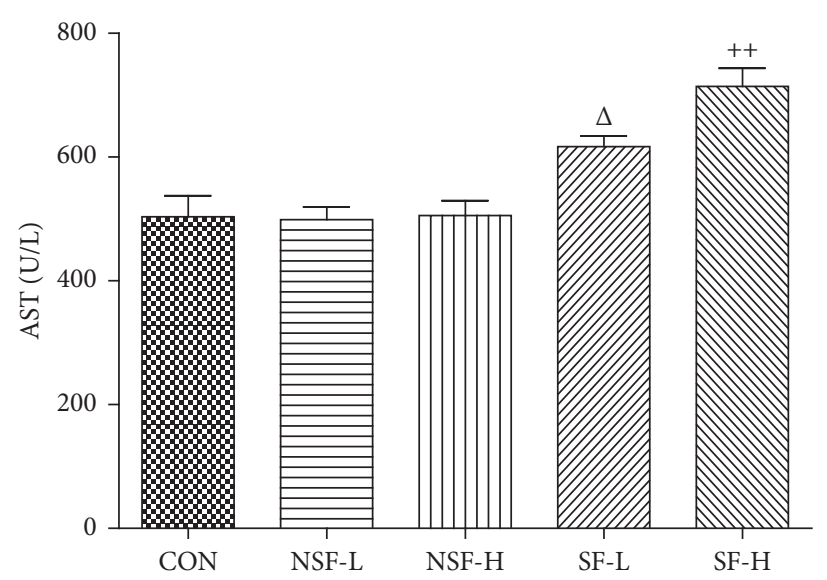

(a)
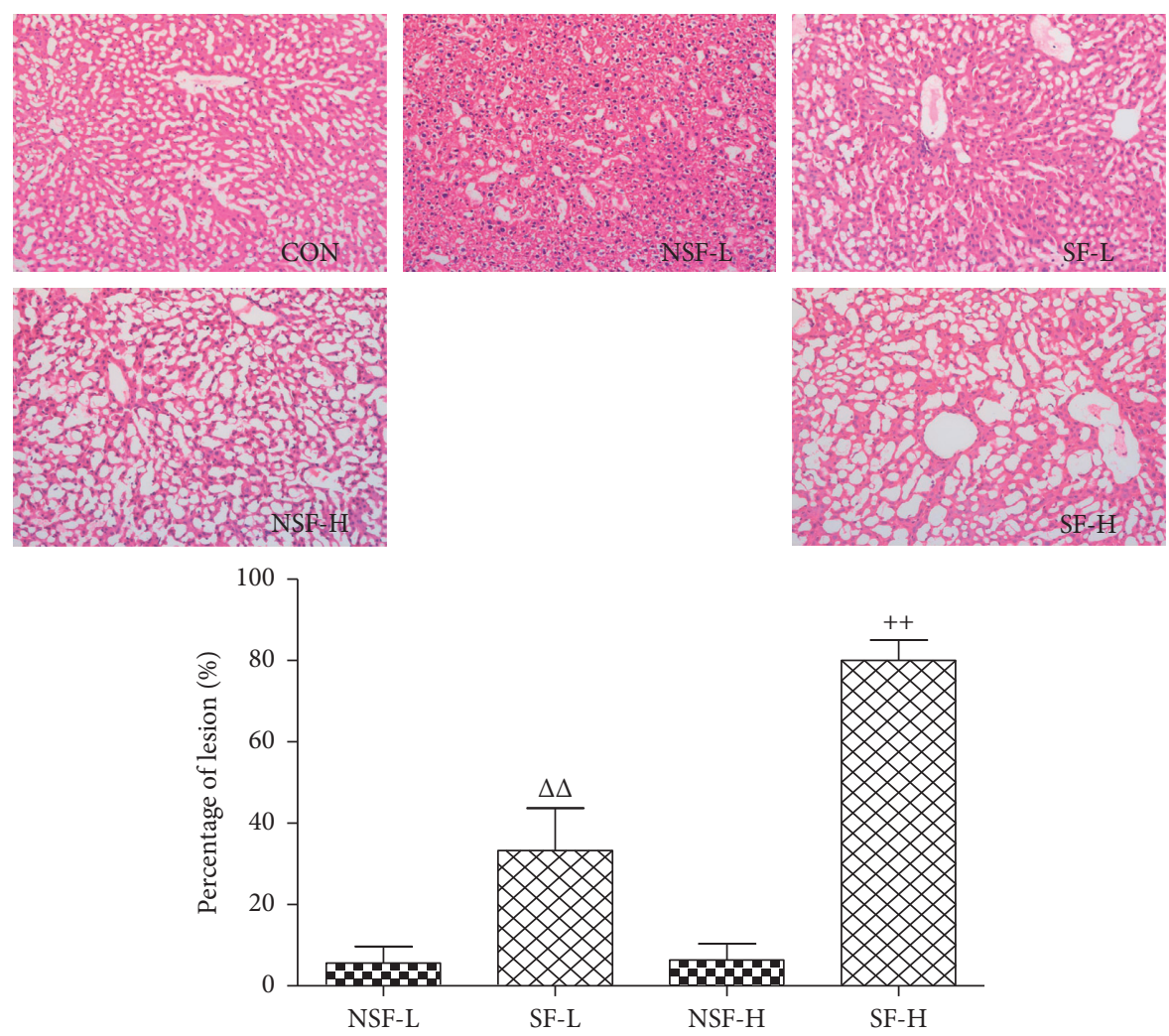

(b)

FIGURE 3: Effects of SF-GRER on liver function in healthy mice $(\bar{x} \pm \mathrm{SD}, n=10)$ : (a) effect of sulfur-fumigated GRER on the serum AST level in healthy mice; (b) representative photomicrographs $(\times 100)$ and lesion assessment of livers after prevention of NSF-GRER and SF-GRER. ${ }^{\Delta}$ compared with NSF-L, $P<0.05 ;{ }^{\Delta \Delta}$ compared with NSF-L, $P<0.01 ;{ }^{++}$compared with NSF-H, $P<0.01$.

products $(m / z, 631.13)$ also had been screened out in SFGRER (Table 3 and Figure 4). It is interesting that LA and ILA had same fragmentation ions $(\mathrm{m} / z, 256.01$ and 418.23) and extremely close retention time (2.44 and $2.46 \mathrm{~min}$ ) because they were isomers. Sulfonation increased the polarity of LA and ILA, so retention time of their sulfonates was advanced to 0.86 and $0.87 \mathrm{~min}$, respectively. The pyrolysis process of LA and ILA in mass spectrometry was first to remove $\beta$-apiose $(\mathrm{m} / z, 418.13$ or 418.39$)$ and then $\beta$-Dglucose $(m / z, 256.07$ or 256.25 , Figure $5(\mathrm{a}))$. The pyrolysis process of $\mathrm{LA}-\mathrm{SO}_{3}$ and ILA-SO $\mathrm{S}_{3}$ in mass spectrometry was to remove sulfonic acid groups $(\mathrm{m} / z, 549.30$ or 549.29, Figure 5(b)). There were no other sulfur-fumigated characteristic products found in such a large amount of screening work.

\section{Discussion}

The advantages to Q-TOF tandem analyzers are their higher mass accuracy and faster scan speeds and duty cycles. However, Orbitrap analyzers do not use magnetic fields to operate, and therefore, cryogenic refrigerants such as liquid 
TABLE 3: UPLC-Orbitrap-MS parameters of characteristic products in SF-GRER.

\begin{tabular}{lcccc}
\hline Name & Retention time $(\mathrm{min})$ & Formula & Precursor ion $(m / z)$ & Mass fragments $(m / z)$ \\
\hline Liquiritin apioside & & & 551.17 & 256.01 \\
& 2.44 & $\mathrm{C}_{26} \mathrm{H}_{30} \mathrm{O}_{13}$ & 254.94 & 418.23 \\
Isoliquiritin apioside & & & & 256.01 \\
& 2.46 & $\mathrm{C}_{26} \mathrm{H}_{30} \mathrm{O}_{13}$ & 551.17 & 257.02 \\
Licuraside & & & & 419.19 \\
& 5.13 & $\mathrm{C}_{26} \mathrm{H}_{30} \mathrm{O}_{13}$ & 551.17 & 441.27 \\
Liquiritin apioside sulfonate & & & & 549.30 \\
Isoliquiritin apioside sulfonate & 0.86 & $\mathrm{C}_{26} \mathrm{H}_{30} \mathrm{O}_{16} \mathrm{~S}$ & 631.13 & 549.29
\end{tabular}

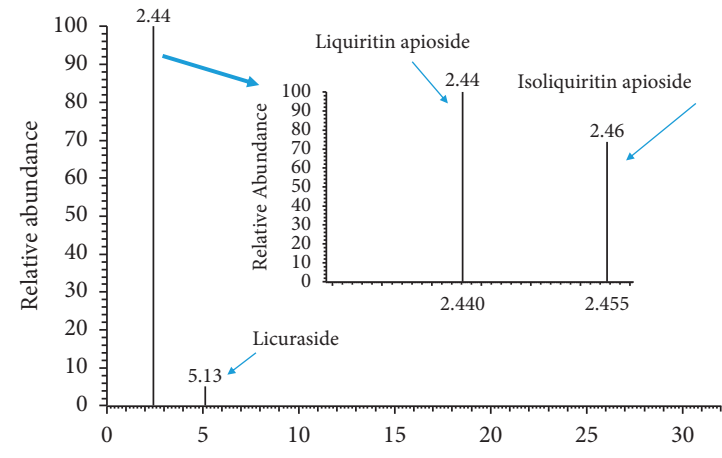

(a)

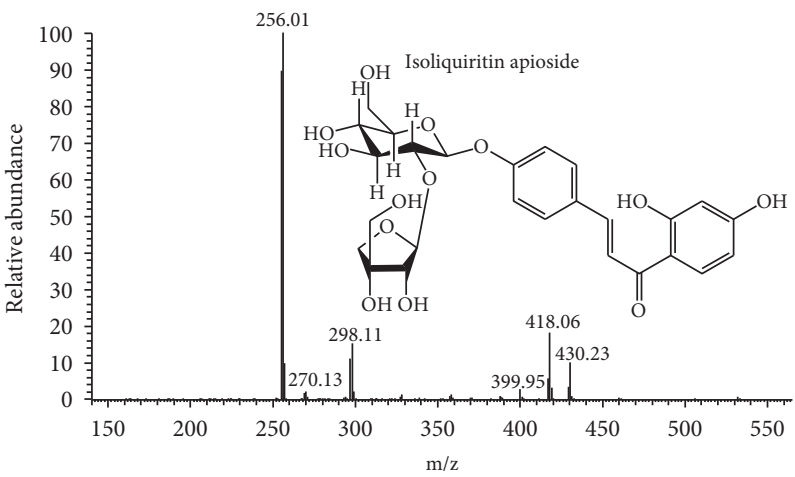

(c)

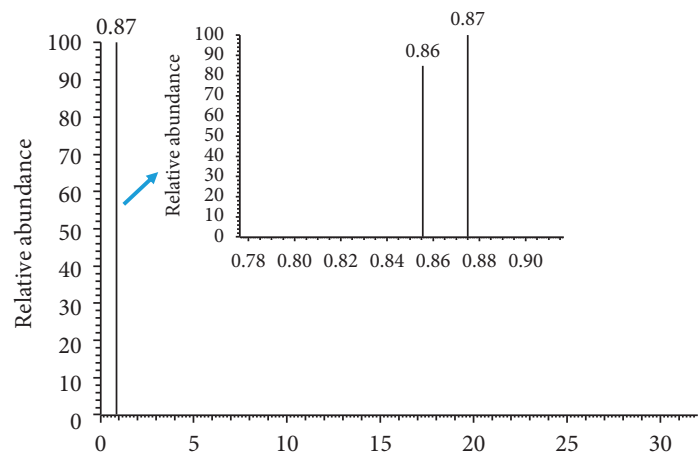

(e)

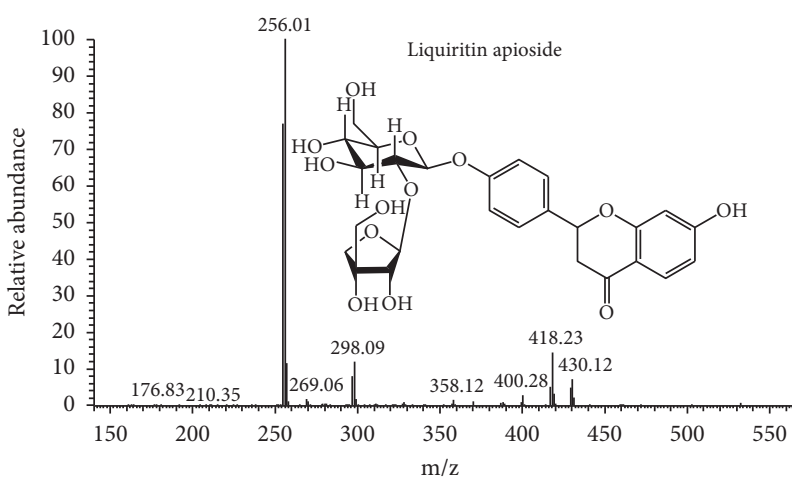

(b)

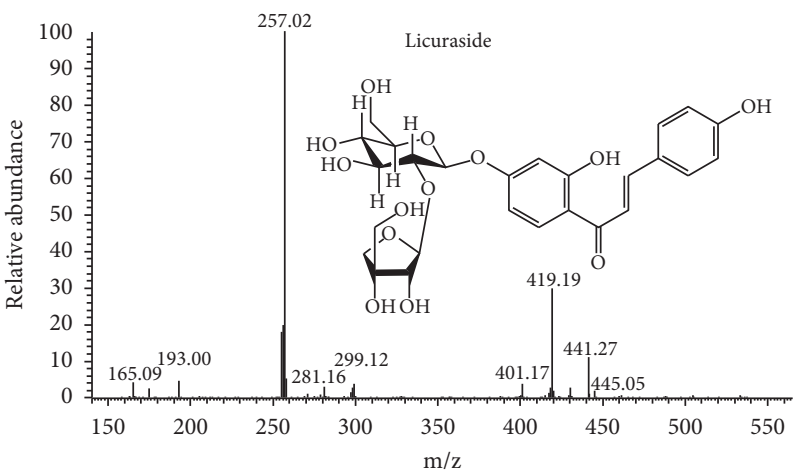

(d)

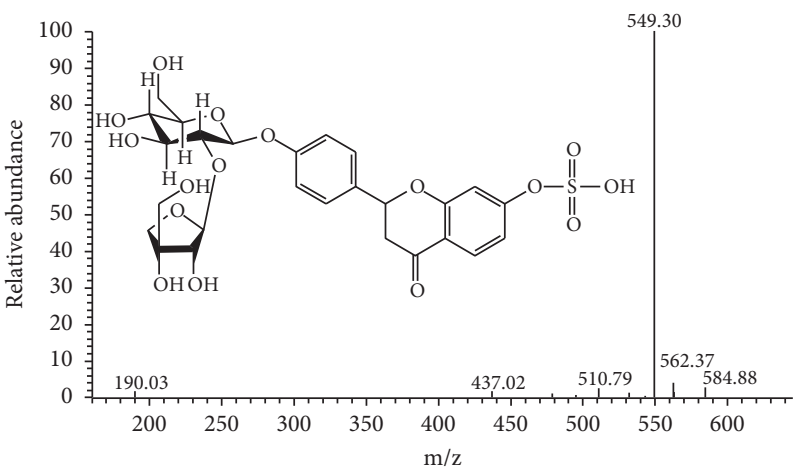

(f)

FIGURE 4: Continued. 


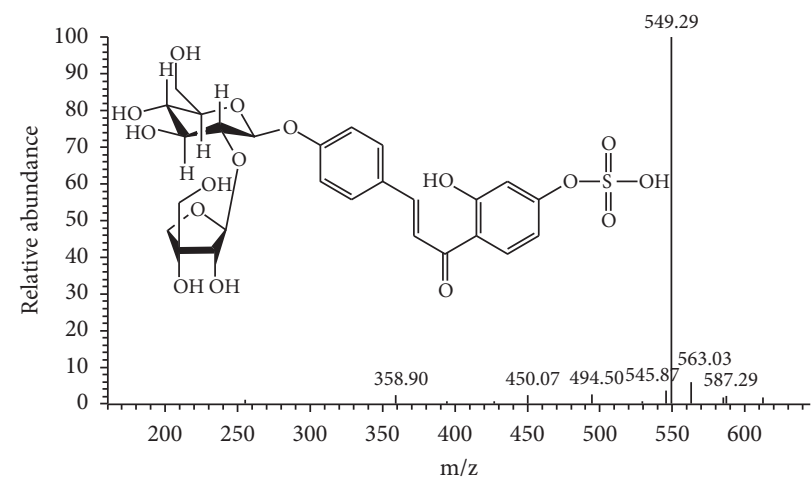

(g)

FIGURE 4: UPLC-Orbitrap-MS spectrum of characteristic products in SF-GRER. (a-d) MS spectrum of liquiritin apioside, isoliquiritin apioside, and licuraside in NSF-GRER. (e-g) MS spectrum of liquiritin apioside sulfonate and isoliquiritin apioside sulfonate in SF-GRER.
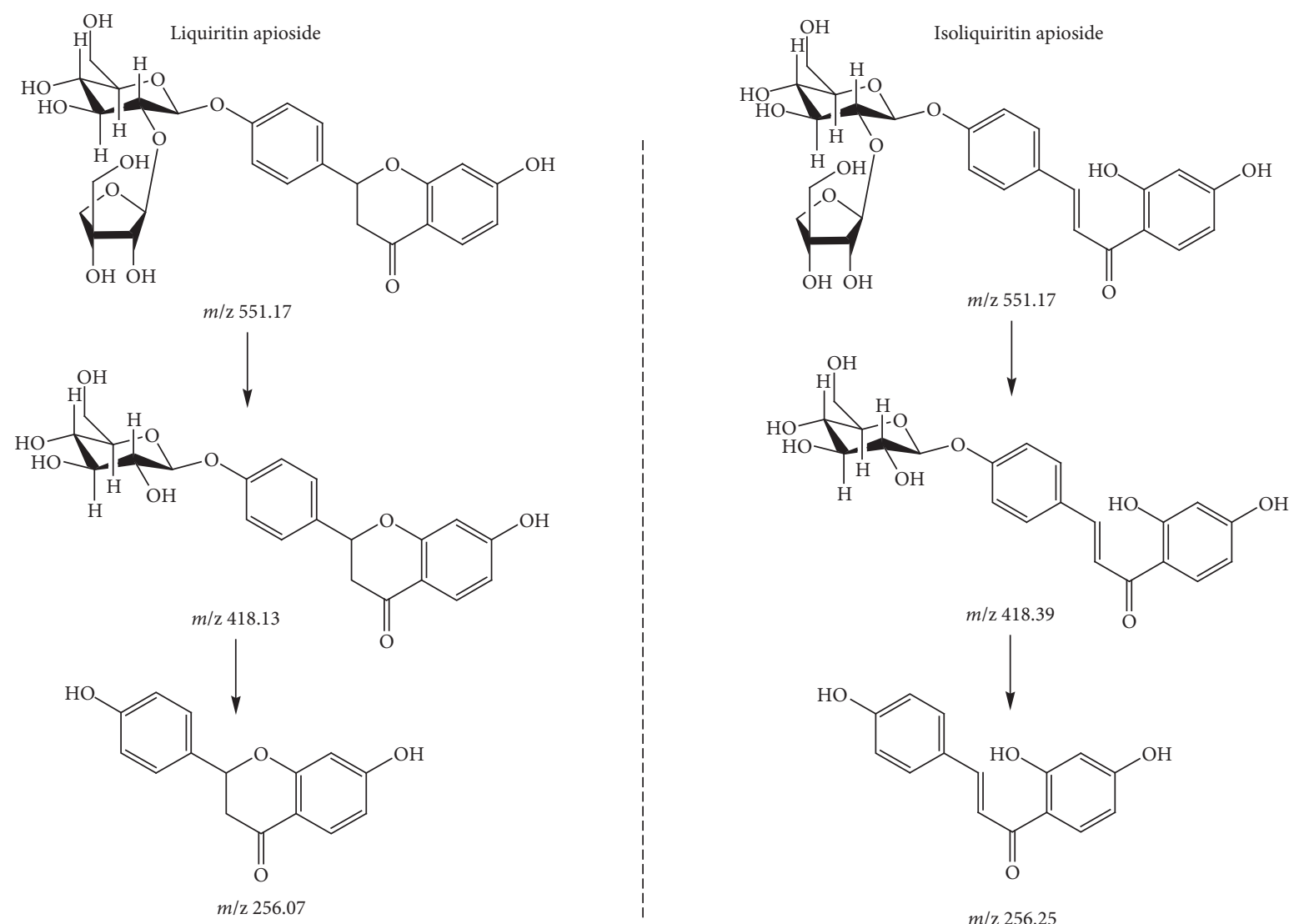

$m / \mathrm{z} 256.25$

(a)

Figure 5: Continued. 

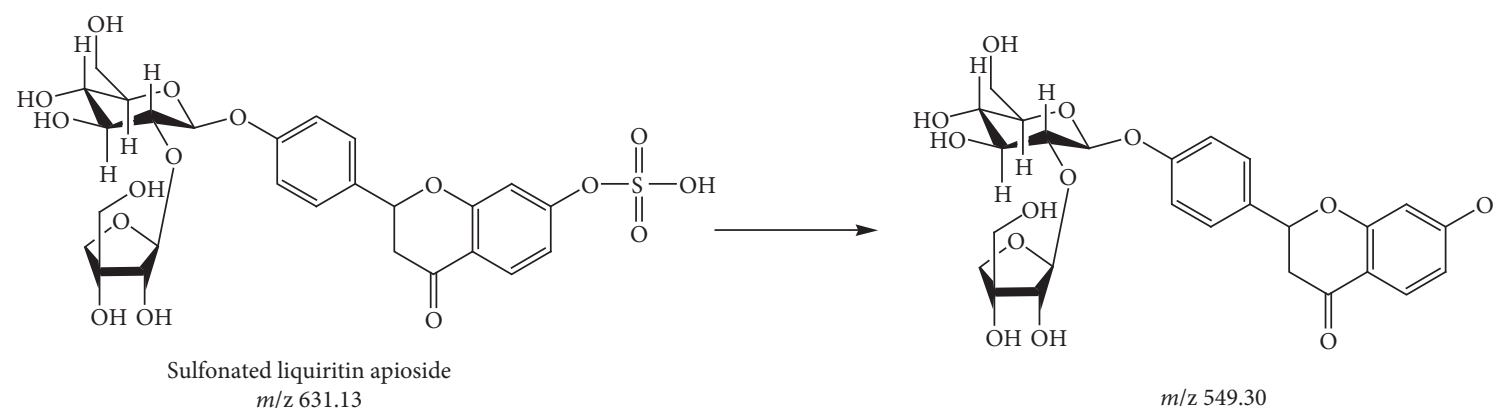

$\mathrm{m} / \mathrm{z} 631.13$
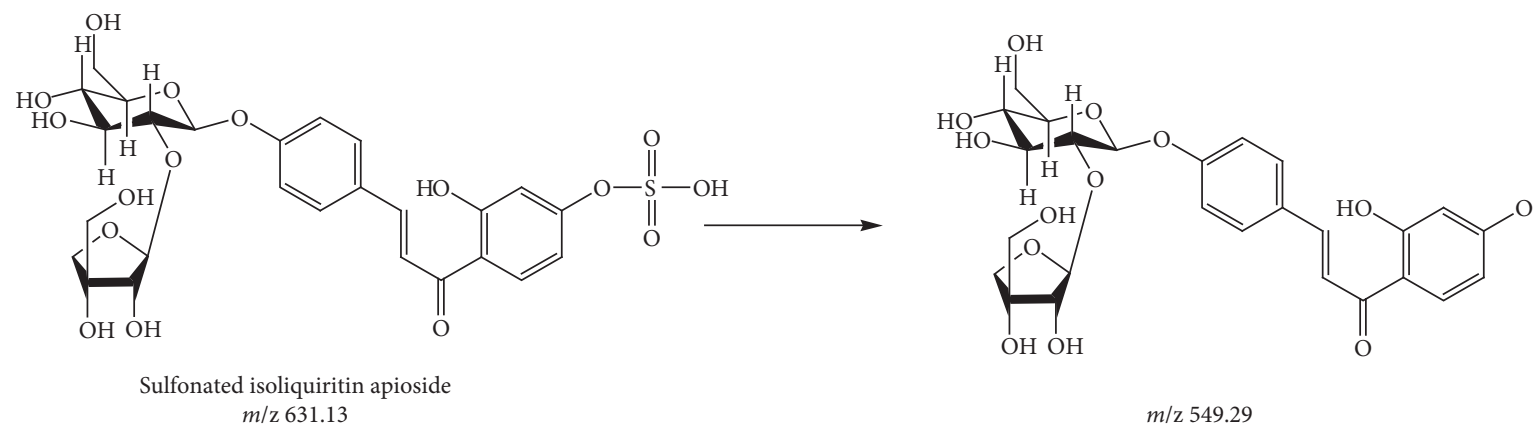
$\mathrm{m} / \mathrm{z} 631.13$

(b)

FIGURE 5: Pyrolysis process of characteristic products in SF-GRER by using UPLC-Orbitrap-MS: (a) pyrolysis of liquiritin apioside and isoliquiritin apioside; (b) pyrolysis of liquiritin apioside sulfonate and isoliquiritin apioside sulfonate.

helium are not necessary and operating costs are kept low. Furthermore, one of the major advantages of the orbitrap analyzer is its high resolving power [35]. Considering these factors, Orbitrap was applied in this paper.

Liquiritin is one of the main active ingredients in GRER which is also an important index for judging the quality of GRER [36]. Pharmacological studies have shown that liquiritin can enhance immune regulation [37]. Therefore, the liquiritin in GRER decreased significantly after sulfur fumigation, which inevitably leads to the decrease of the immune regulation of GRER. Our results also demonstrated that the decrease of liquiritin in GRER after sulfur fumigation was accompanied by a significant decrease in its reversal of immunosuppressive effect induced by cyclophosphamide in mice. Our results confirm that sulfur fumigation damaged the active ingredients in GRER and reduced its pharmacological activity.

More seriously, the level of serum AST and the area of liver lesion increased significantly after continuous administration of sulfur-fumigated GRER in mice. These results suggested that sulfur-fumigated GRER had potential safety hazards, especially increasing the risk of liver injury. Therefore, whether the sulfur fumigation method can be used in GRER is a question worthy of serious consideration. How to effectively identify sulfur-fumigated GRER which has entered the market is also an important technical problem faced by scientific researchers.

In Chinese Pharmacopoeia, the quality control of sulfurfumigated Chinese medicines is only to require the control of $\mathrm{SO}_{2}$ residue less than $400 \mathrm{mg} / \mathrm{kg}$ [38], which is an important indicator to test whether the medicine has been fumigated by sulfur or not (NSF-GRER: $43 \mathrm{mg} / \mathrm{kg}$ and SFGRER, $910 \mathrm{mg} / \mathrm{kg}$ ). However, the residual amount of $\mathrm{SO}_{2}$ is extremely unstable, which will be significantly reduced with the extension of storage time, even lost more than $50 \%$ in 4 months [39]. In order to identify sulfur-fumigated medicinal materials effectively, we suggested that the detection of characteristic products for sulfur-fumigated medicinal materials should be added to the pharmacopoeia. Excitedly, in this study, we successfully used UPLC-Orbitrap-MS technology to screen the characteristic products $(\mathrm{m} / \mathrm{z}$, 631.13) of sulfur-fumigated GRER, which can be applied for the identification of sulfur-fumigated GRER.

\section{Conclusions}

The processing of sulfur fumigation destroyed the active ingredients and attenuated the immunomodulatory effect of GRER. More importantly, the sulfur-fumigated-GRER induced liver damage. In this study, the characteristic products of SF-GRER were first discovered, which provided powerful technical support for its effective identification.

\section{Data Availability}

The data used to support the findings of this study are available from the corresponding author upon request.

\section{Conflicts of Interest}

The authors declare that there are no conflicts of interest regarding the publication of this study. 


\section{Authors' Contributions}

Jun Jiang and Shichang Xiao equally contributed to this work.

\section{Acknowledgments}

This work was supported by the National Natural Science Foundation of China (no. 81703773), Natural Science Foundation of Jiangsu Province (no. BK20170560), and Key R\&D Program Projects in Zhenjiang City (SH2017054).

\section{References}

[1] European Pharmacopoeia 8.0, European Directorate for the Quality of Medicines, Strasbourg, France, 2013.

[2] G. Li, D. Nikolic, and R. B. van Breemen, "Identification and chemical standardization of licorice raw materials and dietary supplements using UHPLC-MS/MS," Journal of Agricultural and Food Chemistry, vol. 64, no. 42, pp. 8062-8070, 2016.

[3] C. Di Lorenzo, A. Ceschi, H. Kupferschmidt et al., "Adverse effects of plant food supplements and botanical preparations: a systematic review with critical evaluation of causality," British Journal of Clinical Pharmacology, vol. 79, no. 4, pp. 578-592, 2015.

[4] P. Montoro, M. Maldini, M. Russo, S. Postorino, S. Piacente, and C. Pizza, "Metabolic profiling of roots of liquorice (Glycyrrhiza glabra) from different geographical areas by ESI/ MS/MS and determination of major metabolites by LC-ESI/ MS and LC-ESI/MS/MS," Journal of Pharmaceutical and Biomedical Analysis, vol. 54, no. 3, pp. 535-544, 2011.

[5] T. Chen, H.-M. Shen, Z.-Y. Deng et al., "A herbal formula, SYKT, reverses doxorubicin-induced myelosuppression and cardiotoxicity by inhibiting ROS-mediated apoptosis," Molecular Medicine Reports, vol. 15, no. 4, pp. 2057-2066, 2017.

[6] H.-S. Kwon, J. H. Park, D. H. Kim et al., "Licochalcone A isolated from licorice suppresses lipopolysaccharide-stimulated inflammatory reactions in RAW264.7 cells and endotoxin shock in mice," Journal of Molecular Medicine, vol. 86, no. 11, pp. 1287-1295, 2008.

[7] R. Rani, S. Dahiya, D. Dhingra, N. Dilbaghi, K.-H. Kim, and S. Kumar, "Evaluation of anti-diabetic activity of glycyrrhizinloaded nanoparticles in nicotinamide-streptozotocin-induced diabetic rats," European Journal of Pharmaceutical Sciences, vol. 106, pp. 220-230, 2017.

[8] Z. Z. Fan, W. H. Zhao, J. Guo et al., "Antidepressant activities of flavonoids from Glycyrrhiza uralensis and its neurogenesis protective effect in rats," Yao Xue Xue Bao, vol. 47, no. 12, pp. 1612-1617, 2012.

[9] Y. Kuang, Y. Lin, K. Li et al., "Screening of hepatoprotective compounds from licorice against carbon tetrachloride and acetaminophen induced HepG2 cells injury," Phytomedicine, vol. 34, pp. 59-66, 2017.

[10] Y. Kuang, B. Li, J. Fan, X. Qiao, and M. Ye, "Antitussive and expectorant activities of licorice and its major compounds," Bioorganic \& Medicinal Chemistry, vol. 26, no. 1, pp. 278-284, 2018.

[11] Y.-H. Ko, S.-H. Kwon, J.-Y. Hwang et al., "The memoryenhancing effects of liquiritigenin by activation of NMDA receptors and the CREB signaling pathway in mice," Biomolecules \& Therapeutics, vol. 26, no. 2, pp. 109-114, 2018.

[12] S. Fujii, O. Morinaga, T. Uto, S. Nomura, and Y. Shoyama, "Development of double eastern blotting for major licorice components, glycyrrhizin and liquiritin for chemical quality control of licorice using anti-glycyrrhizin and anti-liquiritin monoclonal antibodies," Journal of Agricultural and Food Chemistry, vol. 64, no. 5, pp. 1087-1093, 2016.

[13] W. Song, X. Qiao, K. Chen et al., "Biosynthesis-Based quantitative analysis of 151 secondary metabolites of licorice to differentiate medicinal glycyrrhiza species and their hybrids," Analytical Chemistry, vol. 89, no. 5, pp. 3146-3153, 2017.

[14] B. Ma, W. L. T. Kan, H. Zhu, S.-L. Li, and G. Lin, "Sulfur fumigation reducing systemic exposure of ginsenosides and weakening immunomodulatory activity of ginseng," Journal of Ethnopharmacology, vol. 195, pp. 222-230, 2017.

[15] Y. Lou, H. Cai, X. Liu et al., "Multi-component analysis in sun-dried and sulfur-fumigated Angelicae Sinensis Radix by single marker quantitation and chemometric discrimination," Pharmacognosy Magazine, vol. 10, no. Suppl 1, pp. S189-S197, 2014.

[16] L. Chen, A. Kotani, F. Kusu, Z. Wang, J. Zhu, and H. Hakamata, "Quantitative comparison of caffeoylquinic acids and flavonoids in Chrysanthemum morifolium Flowers and their sulfur-fumigated products by three-channel liquid chromatography with electrochemical detection," Chemical and Pharmaceutical Bulletin, vol. 63, no. 1, pp. 25-32, 2015.

[17] S. Dai, Z. Shang, F. Wang et al., "Novelty application of multiomics correlation in the discrimination of sulfur-fumigation and non-sulfur-fumigation ophiopogonis radix," Scientific Reports, vol. 7, no. 1, p. 9971, 2017.

[18] X. Sun, X.-B. Cui, H.-M. Wen et al., "Influence of sulfur fumigation on the chemical profiles of Atractylodes macrocephala Koidz. evaluated by UFLC-QTOF-MS combined with multivariate statistical analysis," Journal of Pharmaceutical and Biomedical Analysis, vol. 141, pp. 19-31, 2017.

[19] Y. Lou, H. Cai, X. Liu et al., "Element analysis and characteristic identification of non-fumigated and sulfur-fumigated Fritillaria thunbergii Miq. using microwave digestion-inductively coupled plasma atomic emission spectrometry combined with Fourier transform infrared spectrometry," Pharmacognosy Magazine, vol. 10, no. Suppl 1, pp. S30-S36, 2014.

[20] M. Kong, H. H. Liu, J. Wu et al., "Effects of sulfur-fumigation on the pharmacokinetics, metabolites and analgesic activity of Radix Paeoniae Alba," Journal of Ethnopharmacology, vol. 212, pp. 95-105, 2018.

[21] M. Yuan, Z. Yan, Y. Liu et al., "Chemical profiles, antioxidant activity and acute toxicity of raw and sulfur-fumigated Smilacis Glabrae Rhizoma," Journal of Ethnopharmacology, vol. 234, pp. 76-84, 2019.

[22] C. Kang, C. J. Lai, D. Zhao et al., "A practical protocol for comprehensive evaluation of sulfur-fumigation of Gastrodia Rhizoma using metabolome and health risk assessment analysis," Journal of Hazardous Materials, vol. 340, pp. 221230, 2017.

[23] W. L. Kan, B. Ma, and G. Lin, "Sulfur fumigation processing of traditional Chinese medicinal herbs: beneficial or detrimental?," Frontiers in Pharmacology, vol. 2, p. 84, 2011.

[24] S. L. Li, H. Shen, L. Y. Zhu et al., "Ultra-high-performance liquid chromatography-quadrupole/time of flight mass spectrometry based chemical profiling approach to rapidly reveal chemical transformation of sulfur-fumigated medicinal herbs, a case study on white ginseng," Journal of Chromatography A, vol. 1231, pp. 31-45, 2012.

[25] J. Jiang, S. Xiao, X. Xu, H. Ma, C. Feng, and X. Jia, "Isomeric flavonoid aglycones derived from Epimedii Folium exerted different intensities in anti-osteoporosis through OPG/RANKL 
protein targets," International Immunopharmacology, vol. 62, pp. 277-286, 2018.

[26] Q. Zhiheng, W. Xiangli, Z. Jinxia, and H. Chenyang, "HighTemperature induced changes of extracellular metabolites in Pleurotus ostreatus and their positive effects on the growth of trichoderma asperellum," Frontiers in Microbiology, vol. 9, p. 10, 2018.

[27] G. Tan, R. Yuan, Z. Hao et al., "Liver function indicators performed better to eliminate cardioembolic stroke than to identify it from stroke subtypes," Journal of Stroke and Cerebrovascular Diseases, vol. 26, no. 1, pp. 230-236, 2017.

[28] X. Meng, X. Chen, L. Wu, and S. Zheng, "The hyperlipidemia caused by overuse of glucocorticoid after liver transplantation and the immune adjustment strategy," Journal of Immunology Research, vol. 2017, Article ID 3149426, 5 pages, 2017.

[29] H. Yang, Y. Shen, Y. Xu et al., "A novel strategy for the discrimination of gelatinous Chinese medicines based on enzymatic digestion followed by nano-flow liquid chromatography in tandem with orbitrap mass spectrum detection," International Journal of Nanomedicine, vol. 10, pp. 49474955, 2015.

[30] N. C. Olivio, B. Julio, R. Jesus et al., "Metabolomic analysis of the Lichen Everniopsis trulla using ultra high performance liquid chromatography-quadrupole-orbitrap mass spectrometry (UHPLC-Q-OT-MS)," Chromatographia, vol. 80, no. 6, pp. 967-973, 2017.

[31] M. Ota, F. Xu, Y. L. Li, M. Y. Shang, T. Makino, and S. Q. Cai, "Comparison of chemical constituents among licorice, roasted licorice, and roasted licorice with honey," Journal of Natural Medicines, vol. 72, no. 1, pp. 80-95, 2018.

[32] M. Huang, Z. Cheng, L. Wang et al., "A targeted strategy to identify untargeted metabolites from in vitro to in vivo: rapid and sensitive metabolites profiling of licorice in rats using ultra-high performance liquid chromatography coupled with triple quadrupole-linear ion trap mass spectrometry," Journal of Chromatography B, vol. 1092, pp. 40-50, 2018.

[33] W. Kong, J. Wen, Y. Yang, F. Qiu, P. Sheng, and M. Yang, "Simultaneous targeted analysis of five active compounds in licorice by ultra-fast liquid chromatography coupled to hybrid linear-ion trap tandem mass spectrometry," The Analyst, vol. 139, no. 8, pp. 1883-1894, 2014.

[34] D. Li, G. Xu, G. Ren, Y. Sun, Y. Huang, and C. Liu, "The application of ultra-high-performance liquid chromatography coupled with a LTQ-Orbitrap mass technique to reveal the dynamic accumulation of secondary metabolites in Licorice under ABA stress," Molecules, vol. 22, no. 10, p. E1742, 2017.

[35] A. M. Haag, "Mass analyzers and mass spectrometers," in Modern Proteomics-Sample Preparation, Analysis and Practical Applications, vol. 919, pp. 157-169, Springer, Advances in Experimental Medicine and Biology, Springer, 2016.

[36] Y. Wei, L. La, L. Wang, R. Batey, C. Wang, and Y. Li, "Paeoniflorin and liquiritin, two major constituents in Chinese herbal formulas used to treat hyperprolactinemia-associated disorders, inhibits prolactin secretion in prolactinoma cells by different mechanisms," Journal of Ethnopharmacology, vol. 204, pp. 36-44, 2017.

[37] N. Ohtake, R. Suzuki, H. Daikuhara et al., "Modulation of lung local immune responses by oral administration of a herbal medicine Sho-saiko-to," International Journal of Immunopharmacology, vol. 22, no. 6, pp. 419-430, 2000.

[38] B. E. Read, Chinese pharmacopoeia (Part IV). Determination of Sulfur Dioxide Residue, Peiping Union Medical College, Beijing, China, 1930.
[39] C. Z. Kang, J. Y. Jiang, W. Z. Yang et al., "Changes of chemical compositions and sulfur dioxide residues of Gastrodiae Rhizoma with different storage times," Zhongguo Zhong Yao Za Zhi, vol. 43, no. 2, pp. 261-266, 2018. 